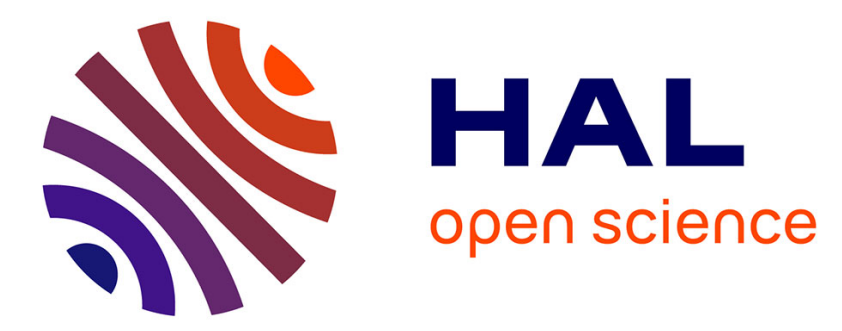

\title{
Dispersion of a Passive Scalar Fluctuating Plume in a Turbulent Boundary Layer. Part III: Stochastic Modelling
}

\author{
Massimo Marro, Pietro Salizzoni, Lionel Soulhac, Massimo Cassiani
}

\section{To cite this version:}

Massimo Marro, Pietro Salizzoni, Lionel Soulhac, Massimo Cassiani. Dispersion of a Passive Scalar Fluctuating Plume in a Turbulent Boundary Layer. Part III: Stochastic Modelling. Boundary-Layer Meteorology, 2018, 167 (3), pp.349 - 369. 10.1007/s10546-017-0330-6 . hal-01925873

\section{HAL Id: hal-01925873 https://hal.science/hal-01925873}

Submitted on 18 Apr 2019

HAL is a multi-disciplinary open access archive for the deposit and dissemination of scientific research documents, whether they are published or not. The documents may come from teaching and research institutions in France or abroad, or from public or private research centers.
L'archive ouverte pluridisciplinaire HAL, est destinée au dépôt et à la diffusion de documents scientifiques de niveau recherche, publiés ou non, émanant des établissements d'enseignement et de recherche français ou étrangers, des laboratoires publics ou privés. 


\title{
Dispersion of a Passive Scalar Fluctuating Plume in a Turbulent Boundary Layer. Part III: Stochastic Modelling.
}

\author{
4 Massimo Marro $^{1}$. Pietro Salizzoni ${ }^{1}$. \\ 5 Lionel Soulhac $^{1} \cdot$ Massimo Cassiani $^{2}$ \\ 7 Received: DD Month YEAR / Accepted: DD Month YEAR
}

\begin{abstract}
We analyze the reliability of the Lagrangian stochastic micromixing method in predicting higher-order statistics of the passive scalar concentration induced by an elevated source (of varying diameter) placed in a turbulent boundary layer. To that purpose we analyze two different modelling approaches by testing their results against the wind-tunnel measurements discussed in Part I (Nironi et al., Boundary-Layer Meteorology, 2015, Vol.156, 415-446). The first is a probability density function (PDF) micromixing model that simulates the effects of the molecular diffusivity on the concentration fluctuations by taking into account the background particles. The second is a new model, named $\operatorname{VP} \Gamma$, conceived in order to minimize the computational costs. This is based on the volumetric particle approach providing estimates of the first two concentration moments with no need for the simulation of the background particles. In this second approach, higher-order moments are computed based on the estimates of these two moments and under the assumption that the concentration PDF is a Gamma distribution. The comparisons concern the spatial distribution of the first four moments of the concentration and the evolution of the PDF along the plume centreline. The novelty of this work is twofold: i) we perform a systematic comparison of the results of micro-mixing Lagrangian models against experiments providing profiles of the first four moments of the concentration within an inhomogeneous and anisotropic turbulent flow, and ii) we show the reliability of the $\operatorname{VP} \Gamma$ model as an operational tool for the prediction of the PDF of the concentration.
\end{abstract}

Keywords Concentration statistics · Fluctuating plume - Gamma distribution • Lagrangian stochastic model · Micromixing modelling

M. Marro

E-mail: marro.massimo@ec-lyon.fr

${ }^{1}$ Laboratoire de Mécanique des Fluides et d'Acoustique, University of Lyon, CNRS UMR 5509 Ecole Centrale de Lyon, INSA Lyon, Université Claude Bernard, 36, avenue Guy de Collongue, 69134 Ecully, France

${ }^{2}$ NILU - Norwegian Institute for Air Research, Kjeller, Norway 


\section{Introduction}

Concentration fluctuations generated by the dispersion of a contaminant from a localized source in a turbulent flow characterize many biological and chemical processes. With fluctuations, we mean here the random behaviour observed for a dispersing scalar in space and time. The full statistical characterization of this random field requires a multi-point, multi-time probability density function (PDF) of the concentration (e.g. Monin and Yaglom, 1975). This is ultimately as complex as fully solving the turbulent flow and it is therefore not feasible. More practically, we may search for the full statistical characterization of the fluctuations in any point of the field, independently from any other point (in space and time), i.e. for the field of the one-point one-time concentration PDF (see e.g. Pope, 2000). Hereafter, we refer to the one-point one-time concentration PDF simply as the concentration PDF.

Common dispersion models based on the Reynolds average concept attempt to characterize the first moment (i.e. the mean) of the concentration PDF only, where the mean is a fundamental property of the PDF. If a process (physical, chemical or biological) has a linear dependence on the concentration, the knowledge of the mean concentration is indeed sufficient to characterize the mean behaviour of this dependent process. However, non-linearity is observed in many cases of practical interest. In such cases, the knowledge of the second and higher moments of the concentration PDF is needed. Particularly relevant cases are the accidental or intentional release of toxic and flammable substances. For example, Gant et al. (2011) relate the likelihood of ignition of a flammable substance to the integral of the concentration PDF between the upper and lower flammability limits at any point. Gant and Kelsey (2012) relate instead the toxic load directly to the integral of the $n-t h$ power of the concentration times the concentration PDF (here $n$ is the toxic load exponent, which Gant and Kelsey (2012) consider equal to two for chlorine and to eight for carbon dioxide). More generally, the knowledge of the concentration PDF is necessary but not sufficient to define the toxic load, which may also require the additional formulation of a model for the correlated concentration time series (e.g. Du et al., 1999; Hilderman and Wilson, 1999; Cassiani et al., 2009). Nonetheless, a necessary starting point, and a significant modelling challenge, is the formulation of a model that is able to forecast the concentration PDF.

To our knowledge, only two modelling methods can be used to directly forecast the concentration PDF at the high Reynolds number characterizing atmospheric flows. These are the transported Lagrangian or Eulerian PDF (micromixing) method (see e.g. Luhar and Sawford, 2005; Cassiani et al., 2005a,b,c, 2007; Garmory et al., 2006; Cassiani et al., 2010; Postma et al., 2011; Amicarelli et al., 2012) and the large-eddy simulation method (see e.g. Henn and Sykes, 1992; Xie et al., 2004). In the latter case, sub-grid-scale concentration fluctuations need to be modelled separately (Colucci et al., 1998) or simply neglected, otherwise the full turbulent flow field would be available at the expense of a formidable computational cost.

All other modelling approaches allow for a direct estimate of two moments of the concentrations only, and rely on assumptions about the form of the concentration PDF. Among these modelling methods, there are the two-particle Lagrangian model (Durbin, 1980; Thomson, 1990; Franzese and Borgas, 2002), the meandering 
plume approach (Gifford, 1959) and its extensions (Yee and Wilson, 2000; Cassiani and Giostra, 2002; Franzese, 2003; Marro et al., 2015), the Eulerian model solving balance equations for the second-order moment (e.g. Milliez and Carissimo, 2008; Yee, 2009), and heuristic Lagrangian methods based on single particle models, e.g. Cassiani (2013).

To our knowledge, the two-particle models are still limited to extremely idealized turbulence conditions, i.e. homogeneous and isotropic turbulence. Applications of the meandering plume approach can be instead extended to the case of non-homogeneous and anisotropic turbulent flows. The meandering model has however two main limitations: i) it is not fully adapted to flows developing in complex geometries, and ii) it requires the setting of several model parameters, including the intensity of the in-plume concentration fluctuations and the functional form of the concentration PDF (e.g. Marro et al., 2015). In this context, second-order closure models have been shown to be more flexible and adapted in forecasting the second moment of concentration also in complex geometries (when the location of interest is not close to the pollutant source), such as those characterizing urban environments (e.g. Milliez and Carissimo, 2008; Yee, 2009). Lastly, recent studies have proposed to extend the use of single-particle Lagrangian models (Thomson, 1987) in heuristic ways, to account for the evolution of the second moment of the concentration fluctuations (Cassiani, 2013; Manor, 2014; Kaplan, 2014). This is the case of the volumetric particle approach (VPA) model that can be viewed as a simplification of a "traditional" Lagrangian PDF micromixing method (Cassiani, 2013). This model is computationally efficient, since it decouples the evolution of the dispersing plume from the background, adopting an approach similar to that used by Alessandrini and Ferrero (2009) for reactive plumes. Different to full Lagrangian PDF micromixing models, this approach requires simulating the trajectories of the marked particles originated at the source only.

The assumptions on the functional form of the PDF (required by the above methods) rely on experimental investigation conducted both in the open field and wind tunnel (a review of these can be found in e.g. Wilson, 1995; Nironi et al., 2015; Oettl and Ferrero, 2017). Here we are particularly concerned with the results presented in Part I (Nironi et al., 2015) and supporting the existence of a universal function for the concentration PDF, as also previously suggested by, e.g., Villermaux and Duplat (2003), Duplat and Villermaux (2008), Yee and Skvortsov (2011), Efthimiou et al. (2016). Nironi et al. (2015) show that the PDFs due to a point source in a turbulent boundary layer are modelled with good accuracy using a family of one-parameter Gamma distributions. Such distributions depend on a single parameter $k$, which is a function of the fluctuation intensity $i_{c}=\sigma_{c} / \bar{c}(\bar{c}$ and $\sigma_{c}$ are the mean and the standard deviation of the concentration, respectively),

$$
p(\chi)=\frac{k^{k}}{\Gamma(k)} \chi^{k-1} \exp (-k \chi)
$$

where $k=i_{c}^{-2}, \Gamma(k)$ is the Gamma function, and $\chi \equiv c / \bar{c}(c$ is the sample space variable).

Herein we compare two micromixing modelling approaches, the transported Lagrangian PDF micromixing model (PMM), adopting an Interaction with the Conditional Mean (IECM) closure, and a new model, named $\operatorname{VP} \Gamma$, based on assuming that the concentration PDF is a Gamma distribution whose two first moments are computed with the volume particle approach (Cassiani, 2013). To that 
purpose we use as a benchmark the experimental data of Nironi et al. (2015), concerning the dispersion in a turbulent boundary layer of a fluctuating plume of passive scalar emitted by an elevated source with two different diameters. As far as we are aware, this represents the first systematic evaluation of this kind of model in the case of inhomogeneous anisotropic turbulence and up to the fourth-order concentration moment.

\section{Model Equations}

The formulation of both models considered here relies on a classical macro-mixing scheme. The temporal evolution of the velocity and position of an ensemble of independent fluid particles is governed by the following stochastic differential equations,

$$
\begin{aligned}
d U_{i}^{\prime} & =a_{i}\left(\mathbf{X}, \mathbf{U}^{\prime}, t\right) d t+b_{i j}\left(\mathbf{X}, \mathbf{U}^{\prime}, t\right) d \xi_{j}, \\
d X_{i} & =\left(\bar{u}_{i}+U_{i}^{\prime}\right) d t,
\end{aligned}
$$

where $a_{i}$ and $b_{i j}$ are the deterministic drift and the stochastic diffusive terms, respectively, $U_{i}^{\prime}$ is the Lagrangian velocity fluctuation, $\bar{u}_{i}$ is the Eulerian mean velocity, $d \xi_{j}$ is an incremental Wiener process (Gardiner, 1983) with zero mean and variance $d t$, and $X_{i}$ is the particle position.

The deterministic acceleration term $a_{i}$ is a function of the turbulent statistics and its three-dimensional formulation is obtained by using the well-mixed condition (Thomson, 1987). With this condition, and assuming a Gaussian velocity PDF with negligible correlations between the different components, the drift term $a_{i}$ is given by

$$
a_{i}=-\frac{U_{i}^{\prime}}{T_{L i}}+\frac{1}{2} \frac{\partial \sigma_{u i}^{2}}{\partial x_{i}}+\frac{U_{i}^{\prime}}{2 \sigma_{u i}^{2}}\left(U_{j} \frac{\partial \sigma_{u i}^{2}}{\partial x_{j}}\right)
$$

with $i=1,2,3$, where $\sigma_{u i}$ represents the r.m.s. velocity of the three components of the Eulerian velocity, i.e. $\sigma_{u}, \sigma_{v}$, and $\sigma_{w}$, and $T_{L i}$ are the Lagrangian integral time scales. These represent the autocorrelation coefficients of the Lagrangian velocity and can be expressed as a function of the velocity variances $\sigma_{u i}^{2}$, the mean turbulent kinetic energy dissipation rate $\varepsilon$, and the Kolmogorov constant $C_{0}$ (Tennekes, 1982) as,

$$
T_{L i}=\frac{2 \sigma_{u i}^{2}}{C_{0} \varepsilon} .
$$

Although this latter relationship was originally obtained for Gaussian homogeneous turbulence, it is widely used in non-homogeneous and even non-Gaussian turbulence conditions (e.g. Luhar and Britter, 1989; Cassiani et al., 2015).

The stochastic diffusive term $b_{i j}$ is defined from the Kolmogorov's hypotheses of self-similarity and local isotropy in the inertial subrange (Obukhov, 1959; Monin and Yaglom, 1975, page 572; Pope, 1987) as,

$$
b_{i j}=\delta_{i j} \sqrt{C_{0} \varepsilon},
$$

where $\delta_{i j}$ is the Kronecker delta. 


\subsection{PDF Micromixing Model (PMM)}

The PDF micromixing model (PMM) aims to solve a transport equation for the concentration PDF explicitly accounting for the dissipative effects of the molecular diffusivity (Pope, 1998). This approach simulates explicitly the micromixing process as given by a mass exchange between polluted fluid particles and 'clean' particles of ambient air, whose trajectories have therefore to be simulated within the whole domain. For this reason, this approach requires a very large amount of computational resources in order to provide accurate solutions.

The simulation of the higher-order moments of the concentration field requires then the introduction of a Markovian state variable $C$ representing the particle concentration,

$$
\frac{d C}{d t}=\phi\left(C, \mathbf{X}, \mathbf{U}^{\prime}, t\right),
$$

where the drift coefficient $\phi$ is responsible for the dissipation of the scalar variance. The IECM model (Pope, 1998) assumes the following parametrization,

$$
\frac{d C}{d t}=-\frac{C-\overline{C \mid \mathbf{X}, \mathbf{U}}}{\tau_{m}},
$$

where $\overline{C \mid \mathbf{X}, \mathbf{U}}$ is the mean scalar concentration conditioned on the local position and velocity and $\tau_{m}$ represents the time scale of the local mixing, which is driven by relative dispersion, and is defined as a function of local velocity variance, mean turbulent kinetic energy dissipation rate, source size, and particle flight time (Cassiani et al., 2005a). The formulation of the micromixing time scale is briefly reported in the Appendix. This model has the desirable property of leaving unaltered the mean concentration field (Fox, 1996; Pope, 1998; Sawford, 2004) and it has been used to simulate the concentration PDF due to atmospheric dispersion from localized sources (e.g. Cassiani et al., 2005a; Postma et al., 2011).

\subsection{The $\operatorname{VP} \Gamma$ Model}

This modelling approach is based on the use of the VPA model and the assumption that the concentration PDF is given by a Gamma distribution. The VPA model was developed by Cassiani (2013) in order to compute the first two moments of the concentration field (mean and variance) without taking into account the background particles. This approach substantially simplifies the representation of the mixing phenomenon and it requires to simulate explicitly only the plume particles. As a consequence, a considerable saving in the computational resources is achieved (see e.g. Fig. 2), and to that purpose, the micromixing process is simulated as a change in a fictitious volume $V_{p}$ associated with the plume particles. The dissipation variance, induced by the molecular diffusivity and driven by the turbulent eddies, is then related to dilution of the marked particles, i.e. an increase of $V_{p}$. To define the volume $V_{p}$ we introduce the mass of tracer $m_{p}$ carried by a particle, and for a non-reactive scalar this mass is conserved $\left(d m_{p} / d t=0\right)$ so that $V_{p}=m_{p} / C$. The temporal evolution of the volume $V_{p}$ is then computed as

$$
V_{p}(t+d t)=V_{p}(t) \frac{C(t)}{C(t+d t)},
$$


where the concentration $C$ is modelled through Eq. 7, by implementing the Interaction by Exchange with the Mean (IEM) model (i.e. with Eq. 8 but adopting a unique velocity class, e.g. Pope, 2000),

$$
\frac{d C}{d t}=-\frac{C-\overline{C(\mathbf{X})}}{\tau_{m}},
$$

where $\overline{C(\mathbf{X})}=\bar{c}$ is the mean concentration in the space domain and $\tau_{m}$ has the same significance and formulation of that discussed in Sect. 2.1 for the IECM model (see Eq. 8 and the Appendix). The computation of $\bar{c}$ requires the spatial discretization of the computational domain and depends on the global mass $M_{c}$ in each space element,

$$
\bar{c}=\frac{M_{c}}{V_{c}}=\frac{1}{V_{c}} \sum_{i=1}^{N_{c}} m_{p_{i}}=\sum_{i=1}^{N_{c}} C_{i} \frac{V_{p_{i}}}{V_{c}},
$$

where $N_{c}$ is the particle number held in the generic cell of volume $V_{c}$. It is worth noting that the use of the IEM model in Eq. 11 does not alter the mean concentration field in the VPA framework (Cassiani, 2013).

The term $V_{p_{i}} / V_{c}$ can be seen as the probability that the particle $i$ takes the concentration $C_{i}$, and the second-order moment $\overline{c^{2}}$ is computed as,

$$
\overline{c^{2}}=\sum_{i=1}^{N_{c}} C_{i}^{2} \frac{V_{p_{i}}}{V_{c}} .
$$

The approximations introduced in the VPA model provide reliable estimates of the first- and second-order statistics, but precludes the accuracy in the estimate of higher-order ones (Cassiani, 2013); for this reason, the VPA model cannot describe the correct evolution of the full concentration PDF.

The computation of the higher-order concentration moments $(>2)$ relies here on the experimental finding by Nironi et al. (2015) about the form of the onepoint concentration PDFs, which was shown to be reliably modelled by a family of one-parameter Gamma distributions (Eq. 1). Assuming a Gamma distribution, the higher-order statistics can then be computed, based on the estimates of the two first moments provided by the VPA model (Eqs. 11 and 12).

\section{Numerical Code}

The Lagrangian stochastic model (Eqs. 2 and 3) and the micromixing models PMM and VPA are implemented in a numerical code using a dynamical expanding grid to minimize the computational resources while maintaining a good accuracy of the numerical solutions (Cassiani et al., 2005a). This approach consists in initially generating a structured grid around the source. During the simulation the grid is advected by the mean field, and expands around the plume as the plume grows. The cell-size expansion is determined by the vertical and transverse plume spreads, while the following boundary conditions are imposed:

- at the top and lateral boundaries, the particle velocity and position are elastically reflected and the concentration is absorbed; 
- at the ground, the particles are elastically reflected and they conserve their concentration.

The elastic reflection of the particles is able to ensure the well-mixed condition (Thomson, 1987) if the turbulence is Gaussian and homogeneous. No simple reflection scheme satisfies the well-mixed condition where the PDF for the normal velocity is asymmetric or locally inhomogeneous (Wilson and Flesch, 1993; Wilson and Sawford, 1996). A general treatment of boundaries ensuring the well-mixed condition is discussed in Thomson and Montgomery (1994). According to Wilson and Flesch (1993), an elastic reflection is acceptable in wall-bounded Gaussian inhomogeneous turbulence, e.g. neutral surface-layer flow.

The micromixing time scales required in the IECM model and IEM model (Eqs. 8 and 10) are estimated during a pre-processing step, computing the trajectories of a smaller ensemble of particles released at the source location, while the mean concentrations are computed on-line during the calculations thus allowing for the straightforward inclusion of chemical reactions (Cassiani et al., 2005a; Cassiani, 2013).

\section{Model Parameters}

The PMM and VPA models require the setting of some free parameters, whose values are generally obtained by fitting the numerical estimates of the first- and second-order concentration moments to the relative values provided by the experiments (e.g. Postma et al., 2011). These parameters are the Kolmogorov constant $C_{0}$ that influences the Lagrangian integral time scales (and therefore the mean concentration), the initial source distribution $\sigma_{0}$, which depends on the source diameter $d_{s}$, the Richardson-Obukhov constant $C_{r}$ and the micromixing constant $\mu_{t}$, which affect the micromixing time (and therefore the concentration variance) The values adopted in the simulations are summarized in Table 1 . The difference

\begin{tabular}{c|c|c|c|c}
$C_{0}$ & $\sigma_{0}$ & $C_{r}$ & $\mu_{t, P M M}$ & $\mu_{t, V P A}$ \\
\hline 4.5 & $\sqrt{2 / 3} d_{s}$ & 0.3 & 0.9 & 0.54
\end{tabular}

Table 1 Model parameter values adopted in the simulations

in the empirical constants of the micromixing time scale $-\mu_{t, P M M}$ and $\mu_{t, V P A}$ - is due to the approximation of the mixing process adopted in the VPA model (Cassiani, 2013). The timestep $\Delta t$ is defined as the minimum among a small fraction of the Lagrangian time scale, the micromixing time scale $\tau_{m}$, and the time scale given by the ratio between grid cell size and $\sigma_{w}$, the standard deviation of the vertical component of the velocity.

The number of velocity classes used in the PMM model is 529 (23 for each of the two spatial directions). In the PMM model the source is represented by marking the particles with a normally distributed scalar concentration $C_{s r c}$,

$$
C_{s r c, P M M}=\frac{Q}{2 \pi \sigma_{0}^{2} \bar{u}_{x}} \exp \left(-\frac{r^{2}}{2 \sigma_{0}^{2}}\right),
$$




\section{Results}
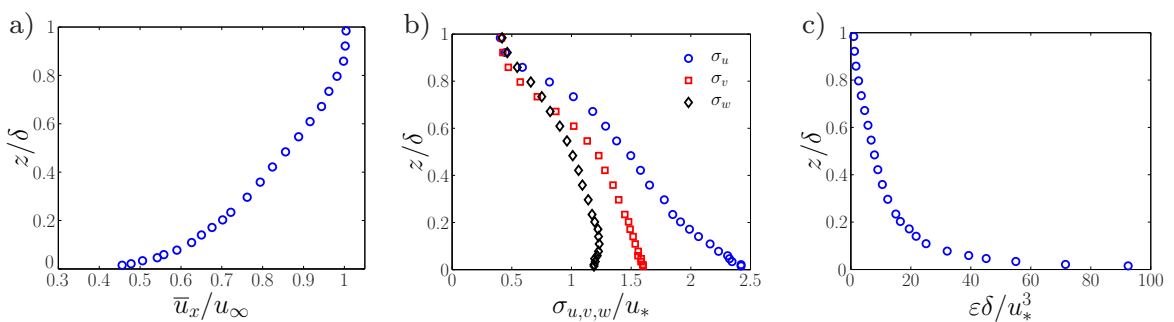

Fig. 1 Vertical profiles of the velocity field imposed in the numerical simulations: a) mean longitudinal velocity $\left.\bar{u}_{x}, \mathrm{~b}\right)$ standard deviations of the three components of the velocity $\sigma_{u}, \sigma_{v}$, and $\left.\sigma_{w}, \mathrm{c}\right)$ turbulent kinetic energy dissipation rate $\varepsilon$. The velocity statistics are normalized using two different velocity scales: the free stream velocity $u_{\infty}$ and the friction velocity $u_{*}$. The ratio between the two is $u_{*} / u_{\infty}=0.037$

We simulated the dispersion of a passive scalar fluctuating plume in the neutral boundary layer and compared the numerical results provided by the two micromixing models - PMM and VP $\Gamma$ - with the wind-tunnel measurements of Nironi et al. (2015). We simulated the continuous releases emitted from an elevated source $\left(z_{s} / \delta=0.19\right)$ of varying diameter $\left.d_{s}: 1\right) \operatorname{ES} 3\left(d_{s}=3 \mathrm{~mm}\right.$, i.e. $\left.d_{s}=0.00375 \delta\right)$, and 2) ES6 $\left(d_{s}=6 \mathrm{~mm}\right.$, i.e. $\left.d_{s}=0.0075 \delta\right)$, where $\delta$ is the boundary layer thickness (equal to $0.8 \mathrm{~m}$ ).

The statistics of the velocity field required as input data for the Lagrangian stochastic models are: the mean longitudinal velocity (Fig. 1a), the standard deviation of the velocity components (Fig. 1b), and the turbulent kinetic energy dissipation rate (Fig. 1c). 


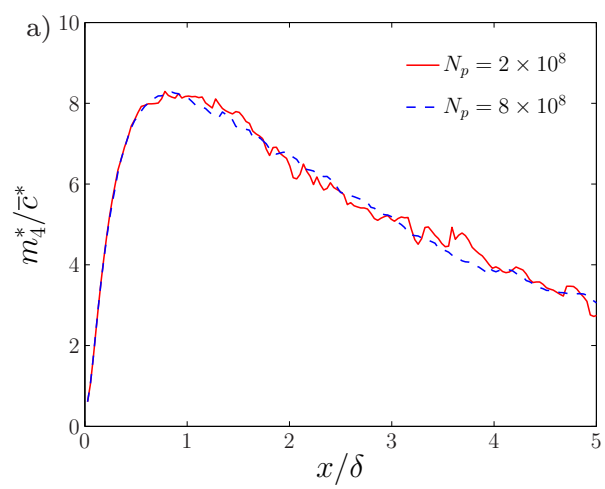

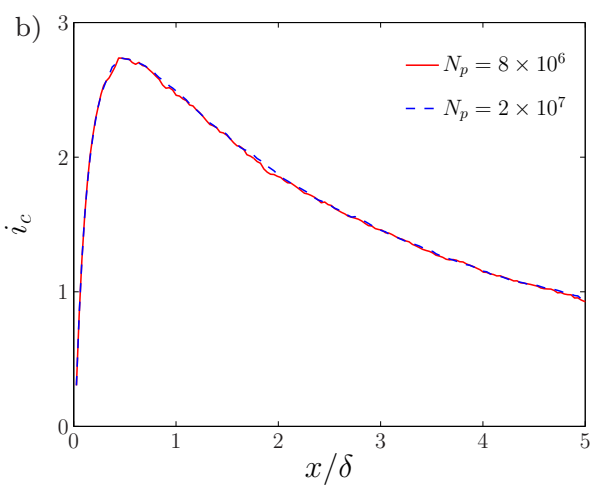

Fig. 2 Influence of the number of particles on the high-order statistics of the concentration vs $x / \delta$ for ES3 at the plume centreline: a) PMM $m_{4}^{*} / \bar{c}^{*}$, b) VPA $i_{c}$

For the PMM model we performed two simulations with varying number of particles $N_{p}, 2 \times 10^{8}$ and $8 \times 10^{8}$, in order to investigate the influence of $N_{p}$ on the accuracy of the numerical solutions. In Fig. 2a we report the longitudinal evolution of the fourth-order moment for the smaller source (ES3). When increasing $N_{p}$, the general tendencies of the numerical solutions as a function of the distance from the source do not vary, even though the profiles become smoother. All the PMM results presented herein were computed using $8 \times 10^{8}$ particles. The sensitivity of the VPA model to the particle number is significantly reduced compared to the PMM model (see Fig. 2b), and very smooth solutions were obtained with $N_{p}=2 \times 10^{7}$. Note that the third- and the fourth-order moments are obtained from the lower moments through the use of the Gamma PDF. Therefore, their longitudinal profiles are as smooth as those of the lower moments.

In order to study the global behaviour of the two models, we focus on the longitudinal profiles of the first four moments of the concentration at the source height $z_{s}$ and at $y=0$. We also focus on the crosswind profiles at two different distances from the source: 1) $x / \delta=0.625$, corresponding to the absolute maximum of the concentration fluctuations, and 2) $x / \delta=3.75$, the position where all concentration statistics become independent of the source size (see Fig. 3). 

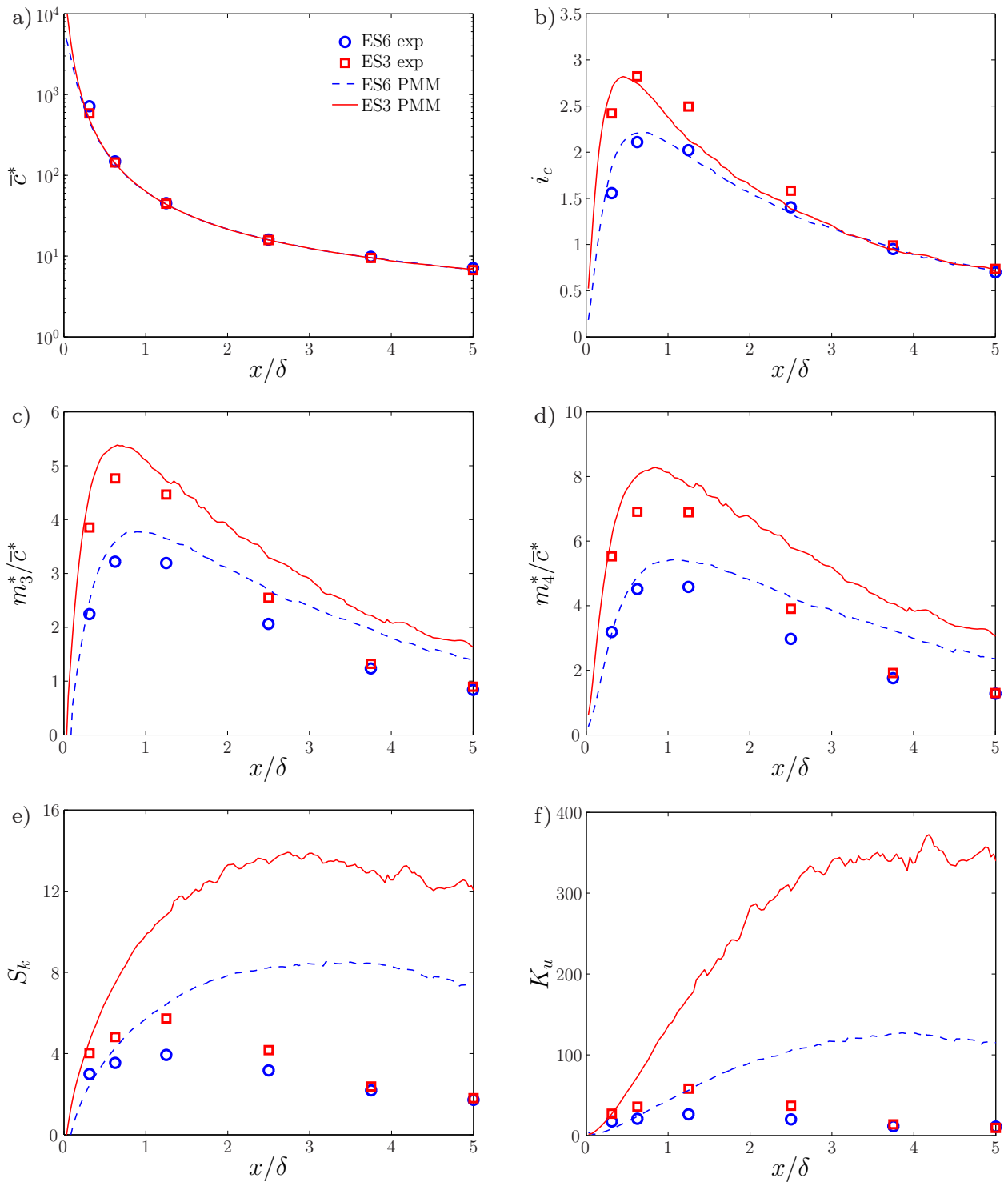

Fig. 3 Results of the PMM model: longitudinal evolution of the concentration statistics: a) normalized mean concentration $\bar{c}^{*}$, b) fluctuation intensity $i_{c}$, c) third-order moment $m_{3}^{*} / \bar{c}^{*}$, d) fourth-order moment $m_{4}^{*} / \bar{c}^{*}$, e) skewness $S_{k}$, f) kurtosis $K_{u}$

The experimental data show that the mean concentration is independent of the source diameter (slight differences can only be detected close to the source location, see Fig. 3a), which instead has a major influence on higher-order moments (Fackrell 

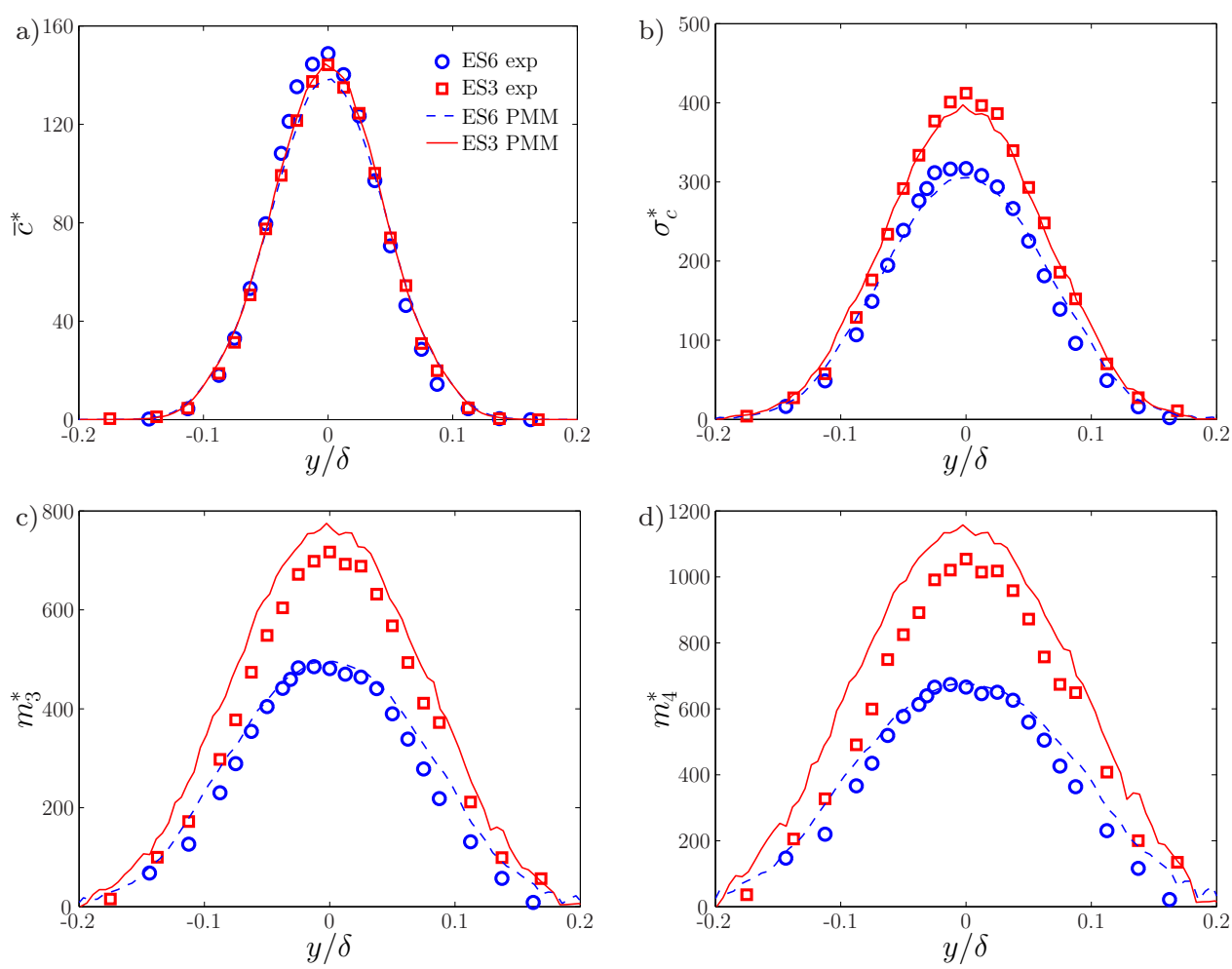

Fig. 4 Results of the PMM model: transverse profiles of the concentration statistics at the source height and $x / \delta=0.625$ : a) $\bar{c}^{*}$, b) $\sigma_{c}^{*}$, c) $m_{3}^{*}$, d) $m_{4}^{*}$

and Robins, 1982; Nironi et al., 2015) up to a distance of approximately $x / \delta=3.75$ from the source (Figs. 3b-d). The comparison between the measurements and the numerical results along the plume centreline in the $x$-direction shows three main features. First, the PMM model is able to compute very accurate solutions for the mean concentration (Fig. 3a). Second, the agreement between experimental and numerical profiles of $i_{c}$ is very satisfactory in all the domain, despite some small differences for the ES3 source at $x / \delta=1.25$ (Fig. 3b). Here, the evaluation of $\sigma_{c}^{*}$ is accurate for the ES6 source, whereas the peak of fluctuations induced by the smallest source is underestimated. Third, even though the values of the $m_{3}$ and $m_{4}$ are reliably simulated in the near field, the model fails in the far field. Here the model significantly overestimates the experimental values and predicts a spurious influence of the source size on $m_{3}$ and $m_{4}$ (Figs. 3c and d).

In Figs. 3e and f we also plot the longitudinal evolution of the skewness $S_{k}$ and the kurtosis $K_{u}$ of the concentration, providing information about the asymmetry and the tails of the PDF. Both parameters are significantly overestimated by the PMM model, which predicts almost constant values in the far field and is not able to reproduce the general tendency given by the experimental data (this aspect will be further investigated in Sect. 5.2).

The experiments show that both $S_{k}$ and $K_{u}$ slightly decrease in the far field, as the PDF concentration seems to slowly tend to a Gaussian distribution. Note 
however that at $x / \delta=5$ the experimental centreline concentration PDF is characterized by $S_{k}=\left(m_{3}^{*} / \sigma_{c}^{*}\right)^{3} \approx 1.7$ and $K_{u}=\left(m_{4}^{*} / \sigma_{c}^{*}\right)^{4} \approx 10$. This shows that, at the end of our domain, the PDF is far from being a Gaussian (which is characterized by $S_{k}=0$ and $\left.K_{u}=3\right)$. The transverse profiles at $x / \delta=0.625$ show
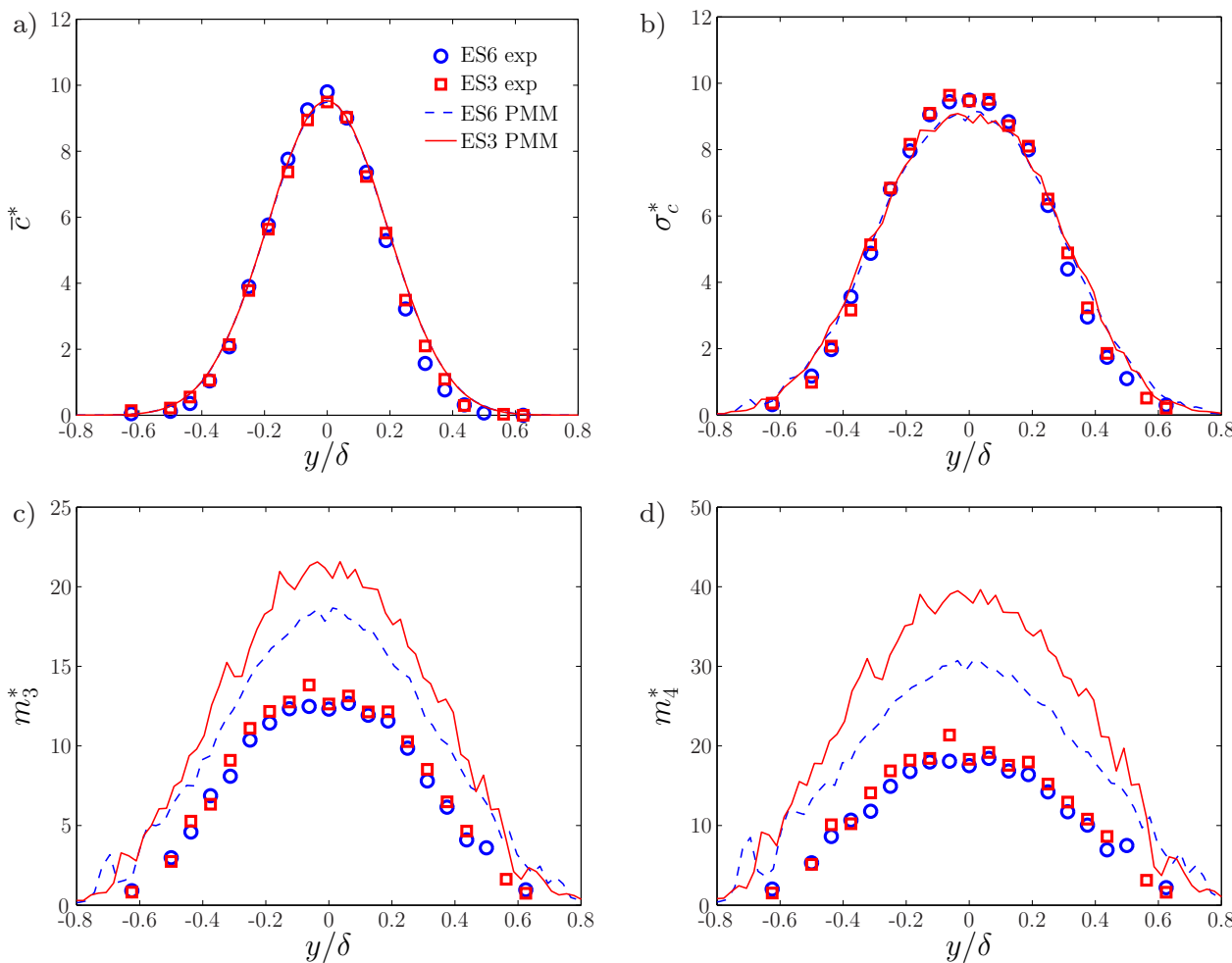

Fig. 5 Results of the PMM model: transverse profiles of the concentration statistics at the source height and $x / \delta=3.75:$ a) $\bar{c}^{*}$, b) $\sigma_{c}^{*}$, c) $m_{3}^{*}$, d) $m_{4}^{*}$

that in the near field the PMM model provides reliable predictions of the first four concentration moments for both sources (Fig. 4). In particular, the accordance for the largest source is very satisfactory, while only a slight overestimate of $m_{3}^{*}$ and $m_{4}^{*}$ is visible for the ES3 source.

As shown by the experiments, at $x / \delta=3.75$, the concentration PDF becomes independent on the source size. This behaviour is correctly reproduced by the model for $\bar{c}^{*}$ and $\sigma_{c}^{*}$, whose profiles are in very good agreement with the experimental data (Figs. 5a and b). As already enlightened by the longitudinal profiles (Fig. 3), the numerical estimates of the third- and fourth-order moments show instead two main problems: i) a significant overestimate of the experimental values, and ii) a persistent influence of the source diameter (Figs. $5 \mathrm{c}$ and d).

We can therefore conclude that the PMM model provides very accurate predictions of the concentration PDFs in the near field. In the far field the PMM model is instead able to reliably simulate the first two moments of the concentration only, 
and fails in reproducing the higher-order moments. Cassiani et al. (2005a) underline that a likely reason for this behaviour is the inability of the IECM (and IEM) deterministic models to correctly relax the concentration PDF in the absence of a mean scalar gradient.

This behaviour is in agreement with what recently observed by Amicarelli et al. (2017) in analyzing the dispersion from a point source in grid turbulence. By comparing the results of an IECM model with experimental data collected at a single downwind position, they found that, despite an optimal matching of the intensity of concentration fluctuations, the skewness and kurtosis were significantly overestimated by the model. As a possible solution to improve the accuracy of these estimates, Amicarelli et al. (2017) suggest increasing the mixing by lowering the value of the micromixing time scale. This implies a slight worsening of the accuracy of the estimate of the concentration fluctuation intensity, while improving that of skewness and kurtosis.

\subsubsection{VPГ Model}

As specified in Sect. 2.2, this approach is based on the VPA model to compute the spatial distribution of the first two moments of the concentration field, i.e. $\bar{c}^{*}$ and $\sigma_{c}^{*}$, and on the assumption that the concentration PDF is a Gamma distribution, i.e. that the third- and the fourth- order moments are given by

$$
\begin{aligned}
& m_{3}^{*}=\left(2 i_{c}\right)^{1 / 3} \sigma_{c}^{*}, \\
& m_{4}^{*}=\left(6 i_{c}^{2}+3\right)^{1 / 4} \sigma_{c}^{*} .
\end{aligned}
$$

Figure 6 shows the longitudinal evolution of the first four-order statistics of the concentration at the plume centreline. The model is able to simulate $\bar{c}^{*}$ and $i_{c}$ in all the domain with good accuracy (Figs. 6a and b), and provides reliable estimates of the higher-order statistics (Figs. 6c and d). In doing this, the $\operatorname{VP} \Gamma$ model is able to reproduce correctly the effects of the source size, including its vanishing influence in the far field. As a consequence the model predicts well also the evolution of both skewness and kurtosis (Figs. 6e and f). Despite this general good agreement between the simulated and measured values, it is still possible to detect some discrepancies between the two. For ES3, the numerical solutions of $i_{c}$ slightly underestimate the experiments in the near field, at $x / \delta=0.625$, and in the intermediate field, at $x / \delta=1.25$.

In the intermediate field, i.e. at $x / \delta=1.25$, the concentration field induced by the ES6 source is very well-reproduced by the model. The computed higher-order moments $\left(m_{3}^{*}\right.$ and $\left.m_{4}^{*}\right)$ of the ES3 source underestimate instead the experimental values. However, the relative error is limited (about $15 \%$ on the centreline). Note also that in the intermediate field the VP $\Gamma$ model tends to slightly underestimate the influence of the source size on the second and the higher-order moments. In the far field, at $x / \delta=3.75$ and $x / \delta=5$, the model values are marginally larger than the experimental ones.

To further investigate these aspects, we focus on the transverse profiles at varying distances from the source. At $x / \delta=0.625$, the numerical profiles of all the moments present a general good agreement with the measurements for both sources (Figs. 7a-d). Only small differences can be found at the peaks of $\sigma_{c}^{*}, m_{3}^{*}$, 

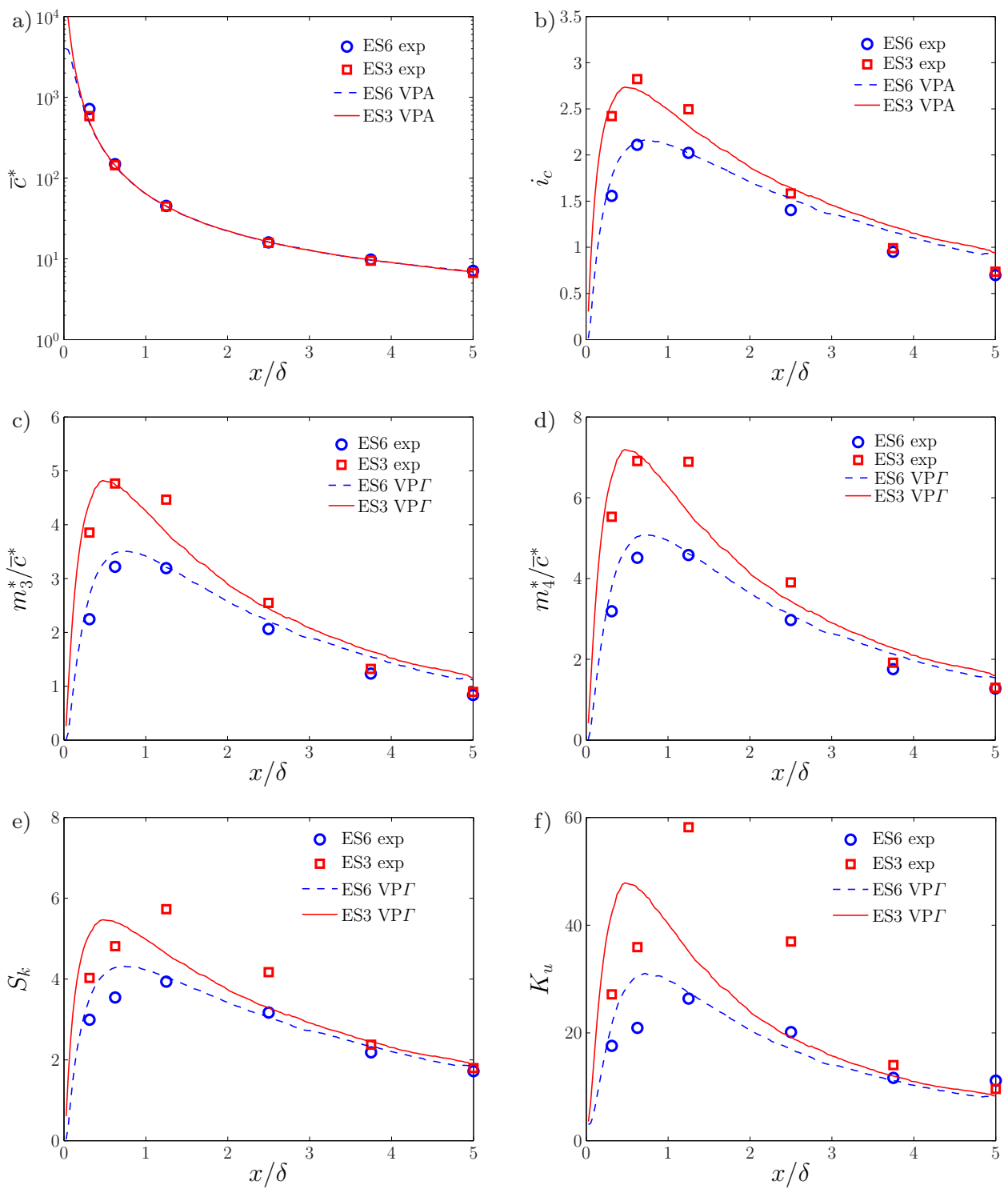

Fig. 6 Results of the $\operatorname{VP} \Gamma$ model: longitudinal evolution of the concentration statistics: a) normalized mean concentration $\bar{c}^{*}$, b) fluctuation intensity $i_{c}$, c) third-order moment $m_{3}^{*} / \bar{c}^{*}$, d) fourth-order moment $m_{4}^{*} / \bar{c}^{*}$, e) skewness $S_{k}$, f) kurtosis $K_{u}$

and $m_{4}^{*}$ produced by ES3 source (we recall that Figs. $7 \mathrm{a}$ and $\mathrm{b}$ represent the output of the VPA model, whereas Figs. $7 \mathrm{c}$ and d were obtained assuming that the PDF is a Gamma distribution). At $x / \delta=3.75$ the experimental data show that the concentration PDFs are independent of the source diameter (Fig. 8). This behaviour is well reproduced by the model. Although the numerical simulations do not give exactly the same values for the two sources, the differences between 

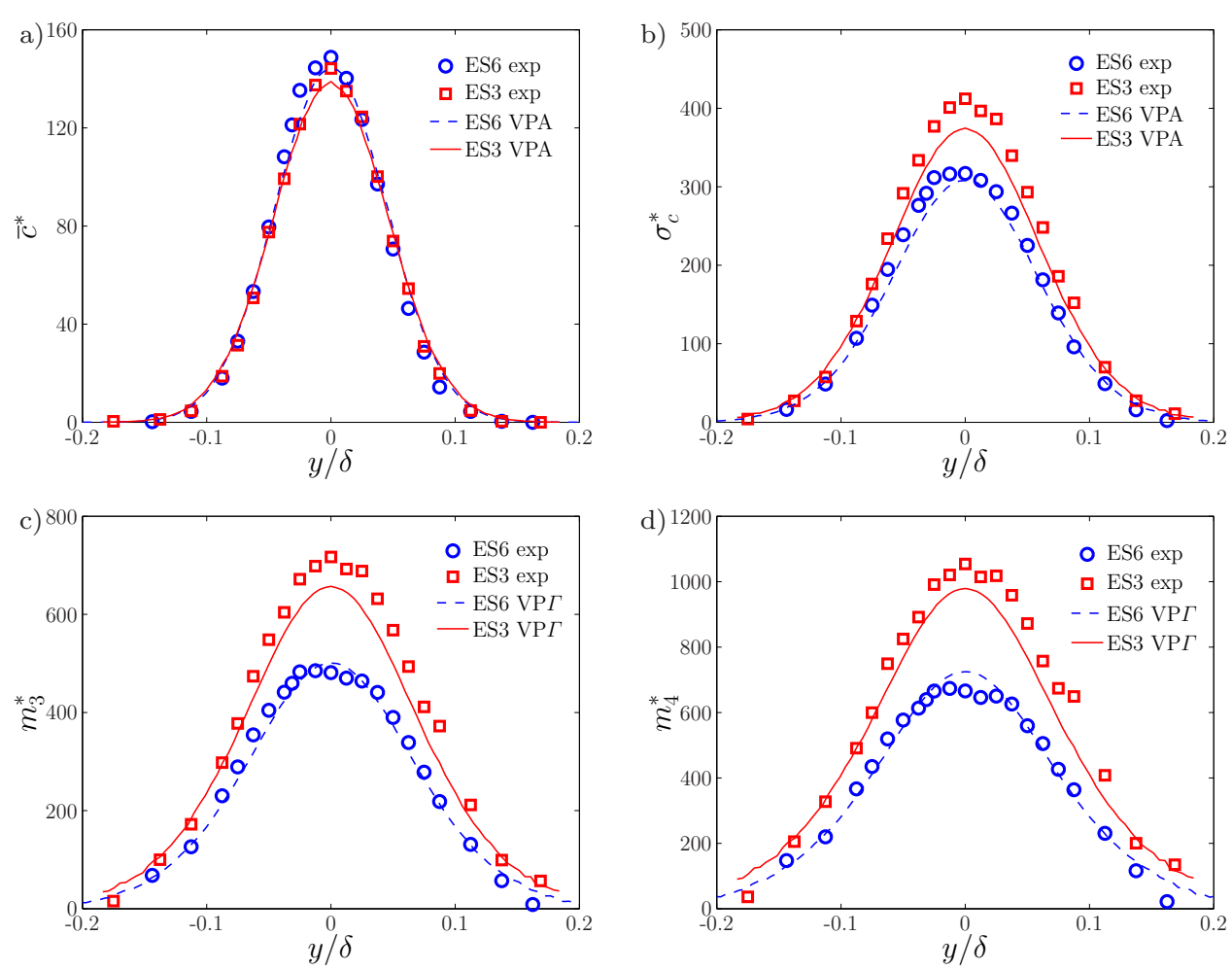

Fig. 7 Results of the $\operatorname{VP} \Gamma$ model: transverse profiles of the concentration statistics at the source height and $x / \delta=0.625$ : a) $\bar{c}^{*}$, b) $\sigma_{c}^{*}$, c) $m_{3}^{*}$, d) $m_{4}^{*}$

the two profiles are very small. Furthermore, the estimates of the concentration statistics are in good agreement with the experiments.

\subsection{One-Point Concentration PDF}

Finally, we turn to the analysis of the concentration PDFs. For the PMM model, the computation of the PDFs are obtained in the classical way: we collect the concentration values carried by a large number of particles in a small control volume and we organize them according to their frequency. For the VP $\Gamma$ model, the shape of the PDF is imposed to be that of a Gamma distribution, completely determined by $\bar{c}$ and $\sigma_{c}$ (see Eq. 1). The PDFs are evaluated at $y=0, z=z_{s}$ and at varying distances from the release point. The PDFs are normalized with the local mean concentration and they are plotted in both linear and logarithmic scale: the linear scale highlights the changes in the PDF shape occurring in the near and the far field (Figs. 9a and b), whereas the logarithmic scale emphasizes how the low frequency values of the sample space variable affect the higher-order moments, namely the skewness and the kurtosis (Figs. 9c and d).

Note that the fluctuating plume considered here is characterized by a large intermittency in the near field, where the dispersion process is dominated by the 

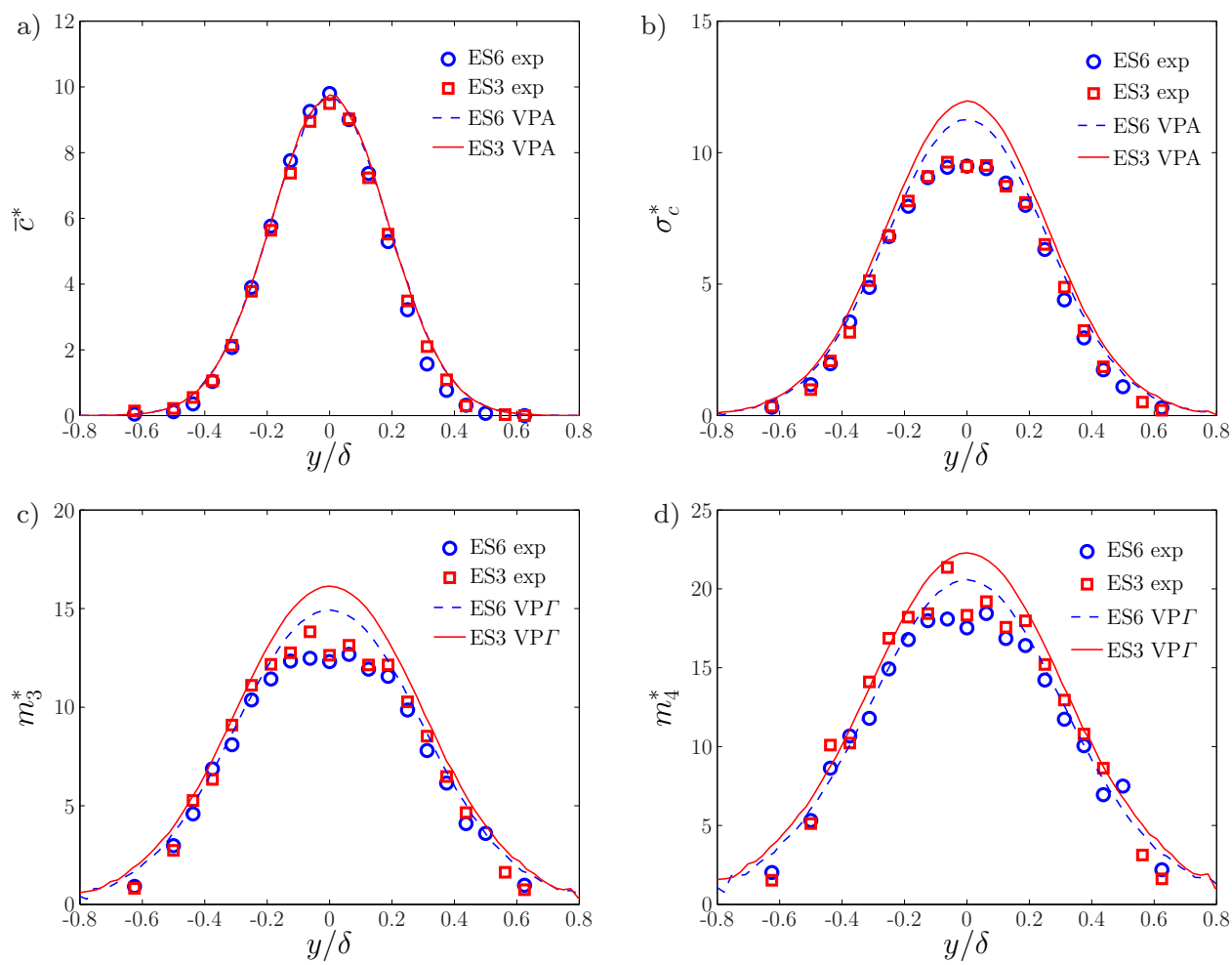

Fig. 8 Results of the $\operatorname{VP} \Gamma$ model: transverse profiles of the concentration statistics at the source height and $x / \delta=3.75:$ a) $\bar{c}^{*}$, b) $\sigma_{c}^{*}$, c) $m_{3}^{*}$, d) $m_{4}^{*}$

meandering (Nironi et al., 2015). In particular, instantaneous concentration measurements show a majority of values very close to zero and few values marked by very high concentration. This implies that the concentration PDF assumes an exponential-like shape (Fig. 9a), that both the models are able to reproduce. Increasing the distance from the source, the influence of the meandering process becomes negligible, the intermittency at the plume centreline reduces and the form of the PDF shifts to a log-normal-like distribution. Fig. 9b shows that, at $x / \delta=5$, both the PMM and VP $\Gamma$ model simulate correctly the experimental data, at least qualitatively.

Further insight into the accuracy of the two micromixing models can be obtained by plotting the concentration PDFs on a logarithmic scale plots (Figs. 9c and d). This helps in evidencing the discrepancies between experimental and modelling results both for low and large values of concentrations. A main indication about the accuracy in the estimates of the PDF is given by the ability to reproduce the values of skewness and kurtosis, providing information about the tails of the PDF (e.g. Heinz, 2003). In order to quantify it, we also compute the following 

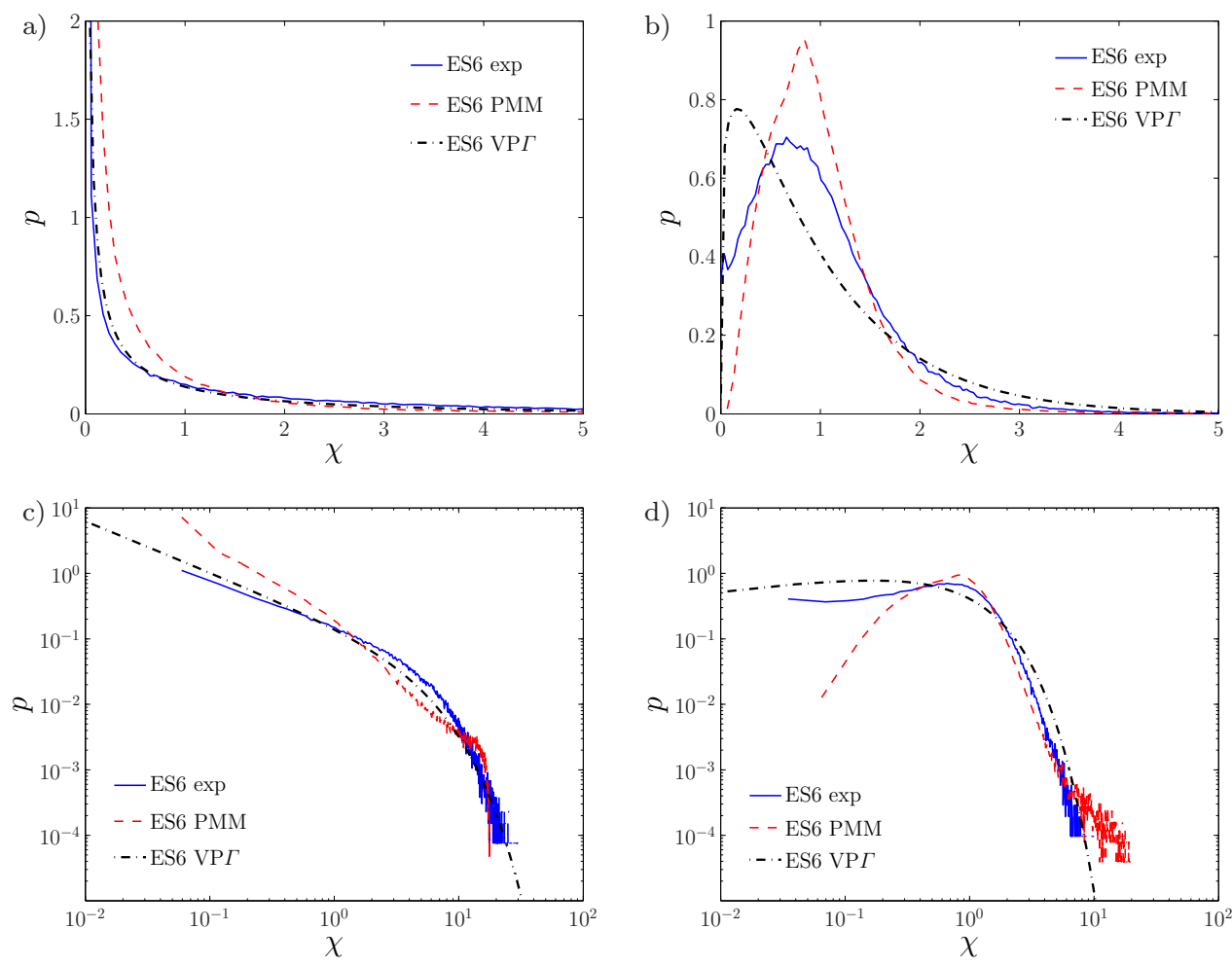

Fig. 9 Concentration PDF of ES6 source at $y=0, z / \delta=z_{s} / \delta$ : a) linear scale at $x / \delta=0.625$, b) linear scale at $x / \delta=5.0$, c) logarithmic scale at $x / \delta=0.625$, d) logarithmic scale at $x / \delta=5.0$

relative errors,

$$
\begin{aligned}
R E_{S_{k}} & =\left|\frac{S_{k_{m o d}}-S_{k_{e x p}}}{S_{k_{e x p}}}\right|, \\
R E_{K_{u}} & =\left|\frac{K_{u_{m o d}}-K_{u_{e x p}}}{K_{u_{e x p}}}\right|,
\end{aligned}
$$

where $S_{k_{e x p}}$ and $K_{u_{e x p}}$ are the experimental values of skewness and kurtosis, respectively, and $S_{k_{m o d}}$ and $K_{u_{m o d}}$ are those estimated numerically.

In Fig. 9c we can observe the three PDFs at $x / \delta=0.625$ in more detail. For low values of $\chi$ we observe some differences between the experimental PDF and that evaluated with the PMM model. Note however that this disagreement does not preclude the model to correctly estimate both the mean and the variance of the PDF (Figs. 3a and b). The relative errors for $S_{k}$ and $K_{u}$ are lower than $21 \%$ $\left(R E_{S_{k}, P M M}=0.206\right.$ and $R E_{K_{u}, P M M}=0.143$, respectively Figs. 10a and b). A similar behaviour is observed for the results of the $\operatorname{VP} \Gamma$ model, where $R E_{S_{k}, V P \Gamma}=$ 0.208 and $R E_{K_{u}, V P \Gamma}=0.457$, even the the relative error of the kurtosis is slight larger than that of the PMM model. The very low relative errors $R e_{K_{u}}$ reveal that in the near field, despite the differences observed in Fig. 9c, both models 

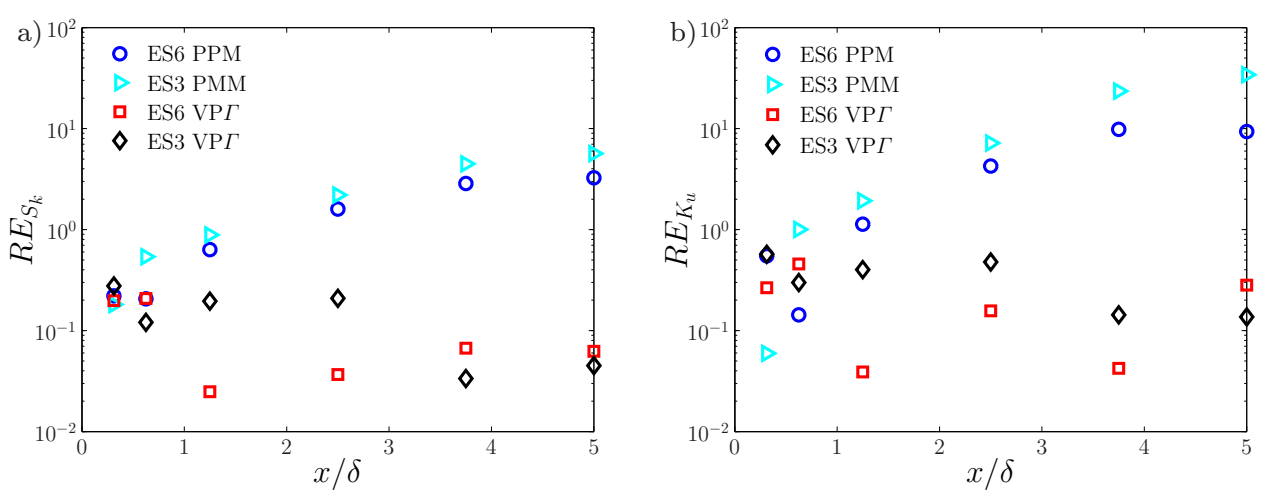

Fig. 10 Longitudinal evolution of the relative error of the skewness and kurtosis at the plume centreline: a) $S_{k}$, b) $K_{u}$

reproduces accurately the complete experimental PDF, including the behaviour of the tails of the distributions.

In the far field the PMM and $\operatorname{VP} \Gamma$ behave differently (Fig. 9d, see also Sect. 5.1.1 and Sect. 5.1.2). The form of the PDF computed with the PMM model suggests that, with respect to the experimental data, the large values of $\chi$ are overestimated and the low values are underestimated. The differences existing between the $\operatorname{VP} \Gamma$ solutions and the measurements are small and the reliability of the model is satisfactory. The magnitude of the relative errors indicates that, for the larger source, the $\mathrm{VP} \Gamma$ relative errors are lower than $30 \%\left(R E_{S_{k}, V P \Gamma}=0.062\right.$ and $R E_{K_{u}, V P \Gamma}=0.282$ ), whereas the PMM model exceed $300 \%$ for the skewness and $900 \%$ for the kurtosis $\left(R E_{S_{k}, P M M}=3.262\right.$ and $R E_{K_{u}, P M M}=9.343$, see also Figs. $10 \mathrm{a}$ and b).

The persistence of the influence of the source size on $m_{3}$ and $m_{4}$ in the far field is an aspect of the PMM model that deserves to be discussed. The experiments show that the two sources - ES3 and ES6 - induce the same concentration field at distances larger than $x / \delta=3.75$ from the release location. The $\operatorname{VP} \Gamma$ model reproduces this feature with good approximation (Fig. 6), whereas the solutions computed by the PMM model exhibit noticeable differences until $x / \delta=5.0$ (Fig. 3).

In Fig. 11 we show the PDFs of the concentration field induced by the two sources (with different size) at $x / \delta=5.0$, as estimated by the experiments and the VP $\Gamma$ model and PMM model simulations. Negligible differences between the sources can be detected for both the experimental data and the $\operatorname{VP} \Gamma$ model simulations (Figs. 11a and b). On the contrary, for the PMM results we observe that the tails of the concentration PDF are quite different for the two source sizes (Fig. 11c). Although the values of the normalized concentration, $\chi$, close to 10 are characterized by very low frequencies $\left(\leq 10^{-3}\right)$, they are responsible for the large discrepancies previously observed between ES6 and ES3 in the computation of $m_{3}$ and $m_{4}$ (see Figs. $3 \mathrm{~b}$ and $\mathrm{c}$ ). 

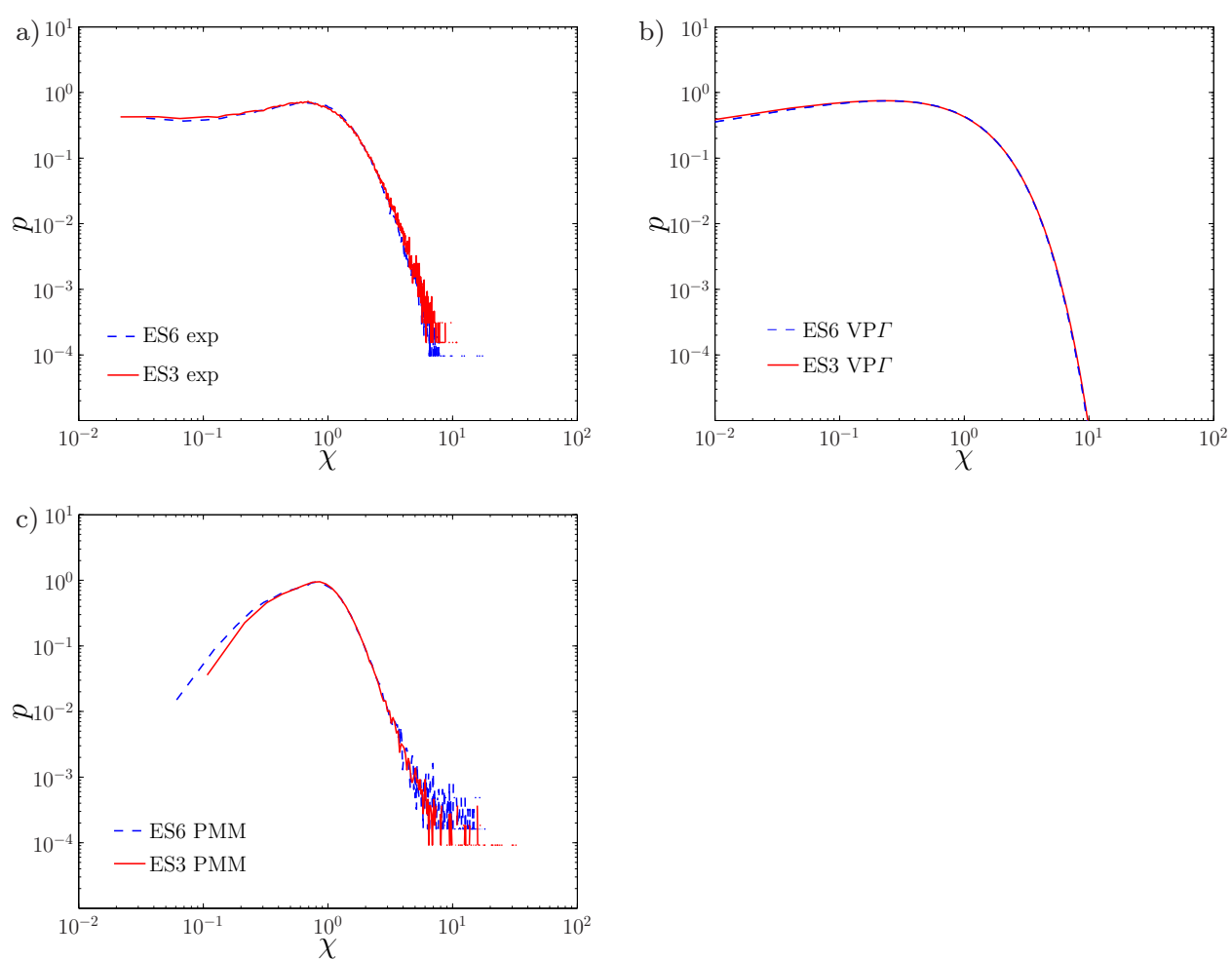

Fig. 11 Concentration PDFs produced by ES6 and ES3 sources at $y=0, z / \delta=z_{s} / \delta$, $x / \delta=5.0$, a) experiments b) VP $\Gamma$ model, c) PMM model

\section{Discussion and Conclusions}

We have tested two micromixing model formulations, the PDF micromixing model (PMM) and the VP $\Gamma$ model and we have investigated their ability in estimating the concentration statistics of a passive scalar emitted within a turbulent boundary layer. The PDF micromixing model (PMM) is a Lagrangian model implementing the IECM closure, for simulating the diffusive mass exchanges between particles. The VP $\Gamma$ model consists in using the volume particle approach (Cassiani, 2013) to compute the first two moments of the concentration and in assuming that the PDF is a Gamma distributions, i.e completely defined by the first two moments (Villermaux and Duplat, 2003; Duplat and Villermaux, 2008; Yee and Skvortsov, 2011; Nironi et al., 2015). The PMM and VPA model were implemented in a Lagrangian stochastic model, using a dynamical expanding grid (Cassiani et al., 2005a).

We simulated the dispersion of a fluctuating plume produced by a continuous release from two point sources of different diameter and we compared the numerical results with the experimental data-set reported in Nironi et al. (2015). The numerical solutions show that the PMM model is able to correctly simulate the concentration statistics in the near field, reproducing effects of the source size on the higher-order moments. In the far field, the numerical and experimental values 
of the mean and standard deviation are in good agreement. Conversely, the values of the modelled third- and fourth-order moments, when compared to the experimental data, show two main limitations of the PMM model. Firstly, the PMM model clearly tends to overestimate the measurements; secondly, the numerical profiles of $m_{3}$ and $m_{4}$ are still sensitive to the size of the source. This is markedly different from what is observed in the experiments (where the source size effects vanishes for $x \geq 3.75 \delta$ ). This behaviour can be reasonably attributed to the inability of the the IECM deterministic model to correctly relax the concentration PDF form towards that of a Gaussian distribution in the absence of a relevant mean scalar gradient (see e.g. Pope, 2000, page 550). Thus overestimating the occurrence of concentration values that are larger than the mean where the mean concentration gradients are weak.

These limitations may be overcome by computing the high-order statistics using the mean and variance, both reliably modelled by the PMM, and assuming that the PDF is a Gamma distribution. We stress that the Gamma distribution hypothesis could be applied to any model providing accurate estimates of the first two moments of the concentration, including e.g. the PMM model, VPA model, higher-order RANS models, etc. Here we chose to calculate the first two concentration moments with the VPA model requiring a number of particles which is significantly smaller than that needed by the PMM model, with a significant saving of memory and computing time. The latter simulation approach, referred to here as the VP $\Gamma$ model, is then suitable for the simulation of dispersion phenomena for operational purposes.

\section{Acknowledgments}

M. Cassiani was partly supported by the European Research Council (ERC) under the European Unions Horizon 2020 research and innovation programme under grant agreement No 670462 (COMTESSA)

\section{Appendix}

We report here the formulation of the micromixing time scale $\tau_{m}$ presented in Cassiani et al. (2005a). In isotropic turbulence $\tau_{m}$ is assumed as depending on the time scale $\tau_{r}$ of the relative dispersion, i.e. the spreading of the plume around its centre of mass,

$$
\tau_{m}=\mu_{t} \tau_{r}=\mu_{t} \frac{\sigma_{r}}{\sigma_{u r}}
$$

\section{where $\mu_{t}$ is an empirical constant to be set, $\sigma_{r}$ is the relative plume spread around} the plume's centroid, and $\sigma_{u r}=\sqrt{\overline{u_{r}^{2}}}$ is the r.m.s of the relative velocity fluctuations. The term $u_{r}$ represents the difference between a turbulent velocity component and the corresponding velocity component of the instantaneous centre of mass (meandering process). We model $\sigma_{r}$ as,

$$
\sigma_{u r}^{2}=\sigma_{u}^{2}\left(\frac{\sigma_{r}}{L}\right)^{2 / 3}
$$


where $\sigma_{u}^{2}$ is the variance of the turbulent velocity, and $L$ represents the Eulerian integral length scale parametrized assuming the stationarity of the energy cascade (Sawford and Stapountzis, 1986),

$$
L=\frac{\left(3 \sigma_{u}^{2} / 2\right)^{3 / 2}}{\varepsilon}
$$

When $\sigma_{r}=L$ the meandering process becomes negligible with respect to the relative dispersion and all the energy contributes to the expansion. For this reason, we imposed the constraint $\sigma_{u r}=\sigma_{u}$, if $\sigma_{r}>L$. We parametrized $\sigma_{r}$ as follows,

$$
\begin{aligned}
\sigma_{r}^{2} & =\frac{d_{r}^{2}}{1+\left(d_{r}^{2}-\sigma_{0}^{2}\right) /\left(\sigma_{0}^{2}+2 \sigma_{u}^{2} T_{L} t\right)}, \\
d_{r}^{2} & =C_{r} \varepsilon\left(t_{0}+t\right)^{3},
\end{aligned}
$$

where $t_{0}=\left(\frac{\sigma_{0}^{2}}{C_{r} \varepsilon}\right)^{1 / 3}$ is the inertial formulation for a dispersion from a finite source size (Franzese, 2003), $\sigma_{0}$ is the source size, and $T_{L}=\frac{2 \sigma_{u}^{2}}{C_{0} \varepsilon}$ is the Lagrangian time scale. Following Cassiani et al. (2005a) the formulation of the micromixing time scale in non-homogeneous and non-isotropic turbulence requires to define the local variance $\sigma_{u}^{2}$ as the average of the variances of the three velocity components. Equation 24 is discretized in time as follows,

$$
\begin{aligned}
& d_{r}^{2}(t+\Delta t)=d_{r}^{2}(t)+3 C_{r} \varepsilon\left(t_{0}+t\right)^{2} \Delta t \\
& d_{r}^{2}(t=0)=\sigma_{0}^{2}
\end{aligned}
$$

where it is worth noting that a Lagrangian stochastic model associated with these micromixing models (PMM and VPA) requires three parameters to be set: $\mu_{t}$, $C_{r}$, and $C_{0}$. The term $C_{0}$ influences the averaged dispersion and its value has to be fixed irrespectively of the used micromixing model (if the micromixing model respects the criterion of not altering the mean concentration field, e.g. Pope, 1998; Sawford, 2004). For this reason, we evaluate $C_{0}$ as the best-fit between the numerical and experimental values of $\bar{c}$ and we found $C_{0}=4.5$. This value is in the range generally accepted in the literature, $2 \leq C_{0} \leq 8$ (Du et al., 1995; Lien and D'Asaro, 2002; Rizza et al., 2006).

The evaluation of $C_{r}$ is performed by comparing the numerical solutions of the concentration variance with the corresponding experimental values. As reported in Table 1, the best-fit is obtained with $C_{r}=0.3$. According to Franzese and Cassiani (2007), $C_{r}$ should be equal to $C_{0} / 11$. Since $C_{0}=4.5$, the value $C_{r}=0.3$ is therefore close to the former theoretical prediction. Finally, $\mu_{t}$ is an empirical constant.

\section{References}

Alessandrini A, Ferrero E (2009) A hybrid Lagrangian-Eulerian particle model for reacting pollutant dispersion in non-homogeneous non-isotropic turbulence. Physica A 388:1375-1387

Amicarelli A, Salizzoni P, Leuzzi G, Monti P, Soulhac L, Cierco FX, Leboeuf F (2012) Sensitivity analysis of a concentration fluctuation model to dissipation rate estimates. Int J Environ Pollut 48:164-173 
Amicarelli A, Leuzzi G, Monti P, Alessandrini S, Ferrero E (2017) A stochastic Lagrangian micromixing model for the dispersion of reactive scalars in turbulent flows: role of concentration fluctuations and improvements to the conserved scalar theory under non-homogeneous conditions. Environ Fluid Mech 17:715753

Cassiani M (2013) The volumetric particle approach for concentration fluctuations and chemical reactions in Lagrangian particle and particle-grid models. Boundary-Layer Meteorol 146:207-233

Cassiani M, Giostra U (2002) A simple and fast model to compute concentration moments in a convective boundary layer. Atmos Environ 36:4717-4724

Cassiani M, Franzese P, Giostra U (2005a) A pdf micromixing model of dispersion for atmospheric flow. Part I: development of the model, application to homogeneous turbulence and neutral boundary layer. Atmos Environ 39:1457-1469

Cassiani M, Franzese P, Giostra U (2005b) A pdf micromixing model of dispersion for atmospheric flow. Part II: application to convective boundary layer. Atmos Environ 39:1471-1479

Cassiani M, Radicchi A, Giostra U (2005c) Probability density function modelling of concentration fluctuation in and above a canopy layer. Agric For Meteorol 133:519-550

Cassiani M, Radicchi A, Albertson JD, Giostra U (2007) An efficient algorithm for scalar pdf modelling in incompressible turbulent flows; numerical analysis with evaluation of iem and iecm micro-mixing models. J Comput Phys 223:519-550

Cassiani M, Franzese P, Albertson J (2009) A coupled Eulerian and Lagrangian mixing model for intermittent concentration time series. Phys Fluids 21:085,105

Cassiani M, Vinuesa JF, Galmarini S, Denby B (2010) Stochastic fields method for sub-grid scale emission heterogeneity in mesoscale atmospheric dispersion models. Atmos Chem Phys 10:267-277

Cassiani M, Stohl A, Brioude J (2015) Lagrangian stochastic modelling of dispersion in the convective boundary layer with skewed turbulence conditions and a vertical density gradient: Formulation and implementation in the Flexpart model. Boundary-Layer Meteorol 154:367-390

Colucci PJ, Jaber FA, Givil P (1998) Filtered density function for large-eddy simulation of turbulent reacting flows. Phys Fluids 10(2):499-515

Du S, Sawford BL, Wilson JD (1995) Estimation of the Kolmogorov constant for the Lagrangian structure function, using a second order Lagrangian model of grid turbulence. Phys Fluids 7:30833090

Du S, Wilson DJ, Yee E (1999) Stochastic time series model for threshold crossing statistics of concentration fluctuations in non-intermittent plumes. BoundaryLayer Meteorol 92:229-241

Duplat J, Villermaux E (2008) Mixing by random stirring in confined mixtures. J Fluid Mech 617:51-86

Durbin PA (1980) A stochastic model of two-particles dispersion and concentration fluctuations in homogeneous turbulence. J Fluid Mech 100:279-302

Efthimiou GC, Andronopoulos S, Tolias I, Venetsanos A (2016) Prediction of the upper tail of concentration distributions of a continuous point source release in urban environments. Environ Fluid Mech 16:1-23

Fackrell JE, Robins AG (1982) The effects of source size on concentration fluctuations in plumes. Boundary-Layer Meteorol 22:335-350 
Fox RO (1996) On velocity conditioned scalar mixing in homogeneous turbulence. Phys Fluids 8:2678-2691

Franzese P (2003) Lagrangian stochastic modeling of a fluctuating plume in the convective boundary layer. Atmos Environ 37:1691-1701

Franzese P, Borgas MS (2002) A simple relative dispersion model for concentration fluctuations in contaminant clouds. J Appl Meteor 41:1101-1111

Franzese P, Cassiani M (2007) A statistical theory of turbulent relative dispersion. J Fluid Mech 571:391-417

Gant S, Kelsey A (2012) Accounting for the effect of concentration fluctuations on toxic load for gaseous releases of carbon dioxide. J Loss Prev Process Ind 25:52-59

Gant S, Pursell M, Lea C, Fletcher J, Rattigan W, Thyer A, Connolly S (2011) Flammability of hydrocarbon and carbon dioxide mixtures. Process Saf Environ Prot 89:472-481

Gardiner CW (1983) Handbook of stochastic methods for physics chemistry and the natural sciences. Springer, New York, USA, 442 pp

Garmory A, Richardson ES, Mastorakos E (2006) Micromixing effects in a reacting plume by the stochastic fields method. Atmos Environ 40:1078091

Gifford F (1959) Statistical properties of a fluctuating plume dispersion model. Adv Geophys 6:117-137

Heinz S (2003) Statistical Mechanics of Turbulent Flows. Springer, Berlin, Heidelberg, Germany, $214 \mathrm{pp}$

Henn DS, Sykes RI (1992) Large-eddy simulation of dispersion in the convective boundary. Atmos Environ 26A:3145-3159

Hilderman T, Wilson DJ (1999) Simulating concentration fluctuation time series with intermittent zero periods and level dependent derivatives. Boundary-Layer Meteorol 91:451-482

Kaplan H (2014) An estimation of a passive scalar variances using a one-particle Lagrangian transport and diffusion model. Physica A 339:1-9

Lien R, D'Asaro EA (2002) The Kolmogorov constant for the Lagrangian velocity spectrum structure function. Phys Fluids 14:4456-4459

Luhar AK, Britter RE (1989) A random walk model for dispersion in inhomogeneous turbulence in convective boundary layer. Atmos Environ 23:1911-1924

Luhar AK, Sawford BL (2005) Micromixing modelling of mean and fluctuating scalar fields in the convective boundary layer. Atmos Environ 39:6673-6685

Manor A (2014) A stochastic single particle Lagrangian model for the concentration fluctuation in a plume dispersing inside an urban canopy. Boundary-Layer Meteorol 150:327-340

Marro M, Nironi C, Salizzoni P, Soulhac L (2015) Dispersion of a passive scalar from a point source in a turbulent boundary layer. Part II: Analytical modelling. Boundary-Layer Meteorol 156:447-469

Milliez M, Carissimo B (2008) Computational fluid dynamical modelling of concentration fluctuations in an idealized urban area. Boundary-Layer Meteorol $127: 241-259$

Monin AS, Yaglom AM (1975) Statistical Fluid Mechanics, vol 2. MIT Press, Cambridge, $874 \mathrm{pp}$

Nironi C, Salizzoni P, Mejan P, Grosjean N, Marro M, Soulhac L (2015) Dispersion of a passive scalar from a point source in a turbulent boundary layer. Part I: Velocity measurements. Boundary-Layer Meteorol 156:415-446 
Obukhov AM (1959) Description of turbulence in terms of Lagrangian variables. Adv Geophys 6:113-115

Oettl D, Ferrero E (2017) A simple model to assess odour hours for regulatory purposes. Atmos Environ 155:162-173, DOI doi: 10.1016/j.atmosenv.2017.02.022.

Pope SB (1987) Consistency conditions for random-walk models of turbulent dispersion. Phys Fluids 30 (8):23742379

Pope SB (1998) The vanishing effect of molecular diffusivity on turbulent dispersion: implications for turbulent mixing and the scalar flux. J Fluid Mech 359:299-312

Pope SB (2000) Turbulent flows. Cambridge University Press, uK, 771 pp

Postma JV, Wilson DJ, Yee E (2011) Comparing two implementations of a micromixing model. part i: wall shear-layer flows. Boundary-Layer Meteorol 140:207-224

Rizza U, Mangia C, Carvalho JC, Anfossi D (2006) Estimation of the Lagrangian velocity structure function constant $c_{0}$ by large-eddy simulation. BoundaryLayer Meteorol 120:25-37

Sawford B (2004) Micro-mixing modelling of scalar fluctuations for plumes in homogeneous turbulence. Flow Turbul Combust 72:133-160

Sawford B, Stapountzis H (1986) Concentration fluctuations according to fluctuating plume models in one and two dimensions. Boundary-Layer Meteorol $37: 89-105$

Tennekes H (1982) Similarity relations, scaling laws and spectral dynamics. In: Niewstadt F, Van Dop H (eds) Atmospheric turbulence and air pollution modelling, D. Reidel Publishing Company, Dordrecht, pp 37-68

Thomson DJ (1987) Criteria for the selection of the stochastic models of particle trajectories in turbulent flows. J Fluid Mech 180:529-556

Thomson DJ (1990) A stochastic model for the motion of particle pairs in isotropic high-reynolds-number turbulence, and its application to the problem of concentration variance. J Fluid Mech 210:113-153

Thomson DJ, Montgomery MR (1994) Reflection boundary conditions for random walk models of dispersion in non-gaussian turbulence. Atmos Environ 28:19811987

Villermaux E, Duplat J (2003) Mixing as an aggregation process. Phys Rev Lett 91:184,501

Wilson DJ (1995) Concentration Fluctuations and Averaging Time in Vapor Clouds. Center for Chemical Process Safety of the American Institute of Chemical Engineers, New York NY

Wilson DJ, Flesch TK (1993) Flow boundaries in random-flight dispersion models: enforcing the well-mixed condition. J Appl Meteorol 32:1695-1707

Wilson DJ, Sawford BL (1996) Review of Lagrangian stochastic models for trajectories in the turbulent atmosphere. Boundary-Layer Meteorol 78:191-210

Xie Z, Hayden P, Voke P, Robins A (2004) Large-eddy simulation of dispersion: Comparison between elevated and ground-level sources. J Turbulence 5:1-16

Yee E (2009) Probability law of concentration in plumes dispersing in an urban area. Environ Fluid Mech 9:389-407

Yee E, Skvortsov A (2011) Scalar fluctuations from a point source in a turbulent boundary layer. Phys Rev E 84:036,306

Yee E, Wilson DJ (2000) A comparison of the detailed structure in dispersing tracer plumes measured in grid-generated turbulence with a meandering plume 
model incorporating internal fluctuations. Boundary-Layer Meteorol 94:253-296 